\title{
Influence of Pilot Pre-Injection Mass on Partially Premixed Dual Fuel (PPDF) Engine Combustion
}

\author{
Abdelrahman Hegab ${ }^{1,2}$, Paul Shayler ${ }^{1}$ \\ ${ }^{1}$ Department of Mechanical, Materials, and Manufacturing Engineering, the University of Nottingham \\ University Park, Nottingham NG7 2RD, United Kingdom \\ Paul.shayler@nottingham.ac.uk \\ ${ }^{2}$ Department of Mechanical Engineering, Al-Azhar University \\ Nasr City, Cairo 11371, Egypt \\ Abdelrahman.hegab@nottingham.ac.uk
}

\begin{abstract}
The development of advanced techniques of diesel engines brings many novel ideas to dual fuel engines, where the key to combustion mode change is essentially the optimization of the pilot injection strategy. Splitting the diesel pilot into a main injection preceded by a pre-injection could bring about a change in the combustion mode from the conventional diesel dual fuel (DDF) combustion to the novel partially premixed dual fuel (PPDF) combustion; if the pre-injection part of the pilot is optimized for timing and quantity. In diesel engines with split injection, the pre-injection part is set to a certain percentage of the total diesel fuel supplied to the engine at the particular operating condition. This could be challenging to PPDF combustion, where the relative proportions of gaseous fuel and diesel could change at the same power level produced. With high levels of gaseous fuel substitution, the mass of diesel pre-injection could drop substantially and potentially lose its effect. The present work aims at investigating the influence of pilot pre-injection mass on PPDF combustion and performance at part load conditions. A comparative study was conducted on a modern automotive direct injection (DI) diesel engine working on PPDF with methane and diesel, between two pre-injection strategies; namely the fixed ratio (FR) and the fixed mass (FM). The tests were performed at different engine speeds ranging from 1400 to $2000 \mathrm{rpm}$ at $25 \%$ of the engine load at the particular speed, with different substitution ratios of methane for diesel fuel (from 20 to $80 \%$ on energy basis). Cylinder pressure, rate of pressure rise (ROPR), coefficient of variation of gross IMEP (COV IMEPgross), heat release rate (HRR), combustion phasing (CA50), brake thermal efficiency, total equivalence ratio, and total diesel fuel injected; are all studied and compared for both pilot pre-injection strategies.
\end{abstract}

Keywords: Dual fuel engine, diesel fuel, methane, partially-premixed, pre-injection mass, combustion, emissions

\section{Introduction}

With recent advances in engine electronic control (EEC) modules and fuel injection equipment (FIE) systems, it has become possible to control fuel injection parameters with ultimate accuracy [1]. This has its implications on gas-diesel dual fuel engines, as the combustion mode in these engines is essentially dependent on pilot injection strategy and its subsequent ignition mode [2]. Conventional DDF combustion mode is attained through using a single injection of the diesel pilot, late in the compression stroke, to act as an ignition source for the premixed gaseous fuel-air mixture. In this mode, the combustion starts with a classical diesel auto-ignition in the pilot region, followed by the combustion of some of the methane-air mixture entrained in the pilot fuel jet and in its immediate vicinity, then flame propagates throughout the rest of methane-air mixture [3]. Yet, within the short time available, flame propagation from the pilot region does not necessarily proceed throughout the entire charge until the concentration of the gaseous fuel exceeds some limiting value (which varies with the fuel employed and operating conditions) [3]. This increases $\mathrm{CO}$ and $\mathrm{HC}$ emissions from the one side, while the intense combustion of the localized pilot promotes the formation of NOx from the other side.

The accurate control of injection parameters enables the attainment of novel combustion modes, such as homogeneous charge compression ignition (HCCI) and partially premixed compression ignition (PPCI) [4]. In HCCI combustion mode, the diesel pilot is injected using a single injection early in the intake stroke, to form a lean homogeneous mixture of dieselmethane-air. If the mixture is perfectly homogeneous, pressure and temperature rise during the compression stroke will lead to a spontaneous ignition, differs from the classical diesel auto-ignition in the sense that it does not occur at a specific 
place in the spray, but simultaneously across the combustion chamber [5]. Reactions in HCCI engines generally involve a two-stage process, including the so-called low temperature and high temperature reactions. For dual-fuel HCCI combustion, the two-stage process is different from those of diesel-like or gasoline-like fuel HCCI combustion and still unclear up to now, but the general features are analogous [2]. The combustion starts with low temperature reactions (LTR) of diesel pilot, or a cool flame zone, that has a high degree of reactivity owing to a high level or radicals [5]. This stage is followed by high temperature reactions (HTR) of the diesel and part of the methane, then the oxidation of the remaining methane takes place at a higher temperature [6]. While the absence of a centralized ignition source and the nonexistence of any rich fuel pockets reduces both NOx and PM substantially, HCCI combustion is very difficult to control as it is ultimately governed by chemical kinetics. It also has a relatively large cyclic variability due to the very lean mixture.

PPCI combustion provides a better and more direct control of combustion phasing, without the need for a closed loop control [7]. In this combustion mode, diesel pilot is split into two injections; an early pre-injection of a comparatively small portion of the pilot, followed by a late injection of the main pilot. The pre-injection acts as an ignition improver; it has a relatively long mixing period with the methane-air mixture at moderate temperature and pressure, where the LTR of diesel fuel at these conditions and the associated radicals promotes the combustion [8]. This mode, therefore, will be referred to as partially premixed dual fuel (PPDF) combustion. The main pilot injection then initiates the combustion that further propagates throughout the cylinder charge, and hence it provides a direct control of combustion timing and phasing.

The determination of the optimum pre-injection quantity in PPDF engines is a delicate issue, as these engines involve the use of varying diesel fuel quantity to accommodate the change in substitution level of the gaseous fuel. If the preinjection part of the pilot is quantified as a percentage of the total diesel fuel used, its mass could drop substantially with high gaseous fuel substitution levels; potentially losing its effect. The present work aims at investigating the influence of pilot pre-injection mass on PPDF Combustion. To achieve that, two approaches of quantifying the pre-injection size will be used; namely the fixed ratio (FR) and the fixed mass (FM). In the FR strategy, the pre-injection is quantified as a percentage of the total diesel fuel used on the specified operating condition (speed and load), and therefore, its mass will decrease with the increase of gaseous fuel substitution level. In the FM strategy, the mass of pre-injection will be held constant at a certain value, and any addition of the gaseous fuel will take place on the account of the main injection part of the pilot. Comparative results between the two strategies will be conducted for cylinder pressure, rate of pressure rise (ROPR), coefficient of variation of the gross IMEP ( $\mathrm{COV}_{\text {IMEPgross }}$ ), heat release rate (HRR), combustion phasing (CA50), brake thermal efficiency, total equivalence ratio, and total diesel fuel injected; at the same operating conditions.

\section{Experimental Facility and Test Conditions}

\subsection{Engine and test bed}

The experimental work in the present study was carried out on a Ford Puma (Duratorq ZSD 422 Range) four cylinder, 2.2 liters, turbocharged intercooled direct injection (DI) diesel engine; typical of that used in commercial light duty vehicles. The engine develops a peak power of $92 \mathrm{~kW}(125 \mathrm{hp})$ at $3700 \mathrm{rpm}$ and a peak torque of $320 \mathrm{Nm}$ at $2000 \mathrm{rpm}$. This engine employs a high-pressure common-rail (HPCR) fuel injection system producing injection pressure as high as 1800 bar. The engine was directly coupled to a David McClure DC electric motor regenerator dynamometer; having a rated power of $90 \mathrm{~kW}$ and a maximum speed of $4000 \mathrm{rpm}$. The dynamometer was controlled through a DC drive unit that can maintain steady speed motoring/absorbing operation against varying torque. The engine intake manifold was properly modified to suite dual fuel operation with port injection of methane. The modification involves attaching a special gas injection system comprising a gaseous fuel rail and a mixing section, where the gaseous fuel is injected and mixed with the intake stream before being admitted to the engine. Chemically pure methane (99.95\% concentration) was supplied from a compressed gas bottle, through a set of pressure regulators to bring the pressure down from the bottle pressure of 200 bar to the injectors working pressure of 7 bar.

\subsection{Data acquisition and instrumentations}

All experimental data were acquired to a desktop PC via an integral data acquisition system and control programs. The setup involves the use of an advanced NIRA engine management system in the place of the original engine control unit (ECU). This system is capable of controlling both the diesel as well as the gaseous fuel injection strategies. It comprises a NIRA-i7r engine control unit complete with NIRA-rk application tool for loading software program, tuning engine data, monitoring, etc. NIRA-i7r ECU is connected through a special dongle to the PC, where NIRA-rk application tool is 
installed. Engine variables, including diesel fuel injection parameters, were measured and controlled by this system. Gaseous fuel injection was also controlled by the NIRA system, while its consumption was measured through a thermal mass flow meter, model F-113AI by Bronkhorst, and then imported into a dedicated software. The in-cylinder pressure was measured using Kistler 6055C piezoelectric non-cooled combustion pressure sensors fitted into the cylinder head glow plug holes. The output signal of each pressure transducer was fed to a dedicated Kistler 5011 charge amplifier. Crankshaft position was monitored using a Hohner W2D11R incremental optical shaft encoder, with an accuracy of 0.5 degree. Incylinder pressure and crank position signals were fed into an X-Series NI USB-6351 high-speed (1MHz) data acquisition module then to the PC, to obtain the crank angle-synchronized in-cylinder pressure data in LabVIEW environment.Type-k thermocouples were used to measure the temperature through the different cycles of the engine (cooling water, oil, intake and exhaust manifolds), and the signals were acquired via a NI-9213 16-channel thermocouple module, mounted on a cDAQ-9171 Compact DAQ chassis connected directly to the PC using a USB cable. In addition, a set of Kulite PT-2028 amplified pressure sensors were placed at different points throughout engine oil circuit and intake and exhaust systems. Figure 1 shows a schematic diagram of the test rig instrumentations and measurement circuits for the engine.

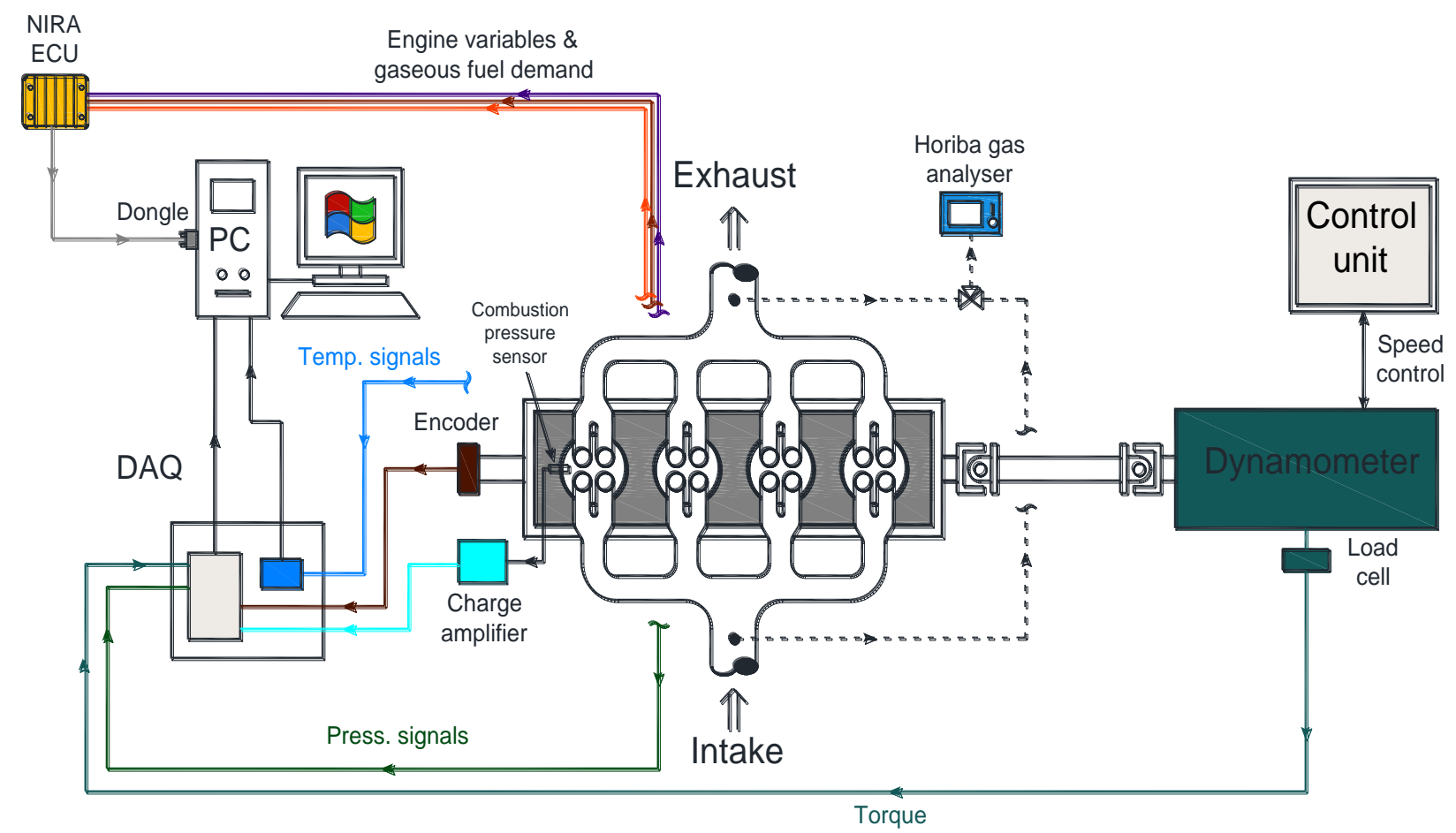

Fig. 1: Schematic diagram of the test rig instrumentations and measurement circuits for the engine.

\subsection{Test conditions and procedure}

The present work targets the operation of methane-diesel dual fuel engines at partial load conditions that prevail in modern city traffic. Accordingly, tests were conducted at different engine speeds ranging from 1400 to $2000 \mathrm{rpm}$ at $25 \%$ of the engine load at the particular speed, with different substitution ratios of diesel fuel to methane ranging from 20 to $80 \%$. The engine was first run under the mono operation of diesel fuel, then methane was introduced on the account of diesel to achieve the required substitution ratio. The percentage of methane substitution is defined on energy basis as:

$$
\% \mathrm{CH}_{4}=\frac{\dot{m}_{C H 4} \times L H V_{C H 4}}{\dot{m}_{D} \times L H V_{D}+\dot{m}_{C H 4} \times L H V_{C H 4}}
$$

where $\left(\dot{m}_{C H 4}\right)$ and $\left(\dot{m}_{D}\right)$ are the mass flow rates of methane and diesel, respectively, and $\left(L H V_{C H 4}\right)$ and $\left(L H V_{D}\right)$ are the lower heating values of the two fuels. As tests involve the use of two fuels, the total equivalence ratio is calculated as: 


$$
\emptyset_{\text {tot }}=\frac{A F R_{C H 4}^{\text {stoic }} \times \dot{m}_{C H 4}+A F R_{D}^{\text {stoic }} \times \dot{m}_{D}}{\dot{m}_{\text {air }}}
$$

where $\left(A F R_{C H 4}^{\text {stoic }}\right)$ and $\left(A F R_{D}^{\text {stoic }}\right)$ are the stoichiometric air-fuel ratios of methane and diesel, respectively, and $\left(\dot{m}_{\text {air }}\right)$ is the mass flow rate of the intake air.

For each test case examined, the variation of cylinder pressure with crank angle position was recorded for 50 consecutive cycles, where the ensemble average of these 50 combustion cycles was then filtered to remove any noise spikes, then processed to calculate HRR, ROPR, CA50, IMEP, and COV IMEPgross. $_{\text {. }}$

The net heat release rate (HRR) is calculated by the traditional first law equation [9]:

$$
\frac{d Q_{n e t}}{d \theta}=\frac{\gamma}{\gamma-1} p \frac{d V}{d \theta}+\frac{1}{\gamma-1} V \frac{d p}{d \theta}
$$

where $(\theta)$ is the crank angle, $(p)$ is the in-cylinder pressure at a given crank angle, $(V)$ is the cylinder volume at that point, and $(\gamma)$ is the specific heat ratio $\left(C_{p} / C_{v}\right)$. Integrating the HRR as a function of crank angle provides a representation of the total energy released up to a specified angle (aka cumulative HR). The crank angle at which $50 \%$ of heat release occurs (CA50) is used to present combustion phasing [10].

The ROPR is obtained from the pressure traces and used as an indication of engine combustion noise [11], while the coefficient of variation of the gross indicated mean effective pressure ( $\left.\mathrm{COV}_{\text {IMEPgross }}\right)$ is used to define the cyclic variability as a measure of engine stability, and calculated as [9]:

$$
\operatorname{COV}_{\text {IMEPgross }}=\frac{\sigma_{I M E P g}}{\overline{I M E P g}} \times 100 \%
$$

where $\left(\sigma_{I M E P g}\right)$ is the standard deviation and $(\overline{I M E P g})$ is the mean value of the gross IMEP over the recorded cycles.

For both fixed ratio (FR) and fixed mass (FM) strategies, the pre-injection timing was held constant @ 50 BTDC, where this value was found to be the optimum value that could achieve the two-stage PPDF combustion. The main injection timing was also kept @ $10^{\circ}$ BTDC. For FR strategy, the size of the pre-injection was quantified as a percentage of the total diesel fuel used:

$$
\% \mathrm{FR}=\frac{\dot{m}_{P r e}}{\dot{m}_{D}}
$$

where $\left(\dot{m}_{\text {Pre }}\right)$ is the mass flow rate of pre-injection part of the pilot, and $\left(\dot{m}_{D}\right)$ is the total mass flow of diesel fuel (supplied to the engine; inclusive of the pre-injection (i.e. any change in the total diesel fuel used when $\% \mathrm{CH}_{4} \mathrm{changes}$ necessarily means the mass of the pre-injection changes). The optimum value of \%FR that was achievable with the current fuel injection equipment (FIE) system throughout the range of test cases, without substantially affecting the cool flame reactions or the ROPR, was found to be $20 \%$. This was determined from the engine operation at conventional diesel mode. Accordingly, it was decided to set the base value of fixed mass strategy (\%FM) to be $20 \%$ of the total mass of the diesel fuel used to operate the engine in conventional diesel operation; at the specified operating conditions. This value was held constant throughout any $\% \mathrm{CH}_{4}$ used, and any change in the total diesel fuel mass was solely addressed by changing the main injection quantity.

\section{Results and Discussion}

Due to space limitation, only test results at $1400 \mathrm{rpm}$ and $25 \%$ of the engine load are presented for PPDF with FR pre-injection (will be referred to as FR-PPDF) and with FM pre-injection (will be referred to as FM-PPDF). This operating point is expected to significantly show the effect of pre-injection mass, due to the limited quantity of total 
diesel fuel used. Figure 2 shows the in-cylinder pressure traces and lists the associated numeric values of maximum ROPR at each combustion mode; at different $\mathrm{CH}_{4}$ substitution ratios. It can be seen from Fig. 2 (a) that the in-cylinder pressure with FR-PPDF combustion considerably drops as the $\% \mathrm{CH}_{4}$ increases; while FM-PPDF combustion always maintains the high levels of cylinder pressure as shown in Fig. 2 (b). This could reveal that with FR pre-injection, the decrease in the preinjection mass as the $\% \mathrm{CH}_{4}$ increases eventually causes the pre-injection to lose its effect in promoting the combustion. As a consequence, the ignition delay (ID) of the main part of the diesel gets prolonged as the $\% \mathrm{CH}_{4}$ increases [12]. The late start of combustion (SOC) implies that the peak cylinder pressure will be attained at a later position relative to the TDC; at lower values. With FM pre-injection, in contrast, the presence of active radicals from the LTR zone promote the combustion process. The earlier start of that intensified combustion yields higher cylinder pressure values [13]. Furthermore, at the increase in $\% \mathrm{CH}_{4}$ takes place on the account of the main injection, the main combustion event will start with an auto-ignition of a less amount of the diesel fuel, and hence the maximum ROPR values are much less; the average reduction in maximum ROPR exceeds $8 \%$. This is advantageous to engine combustion noise, fuel economy, and NOx and PM emissions [11].
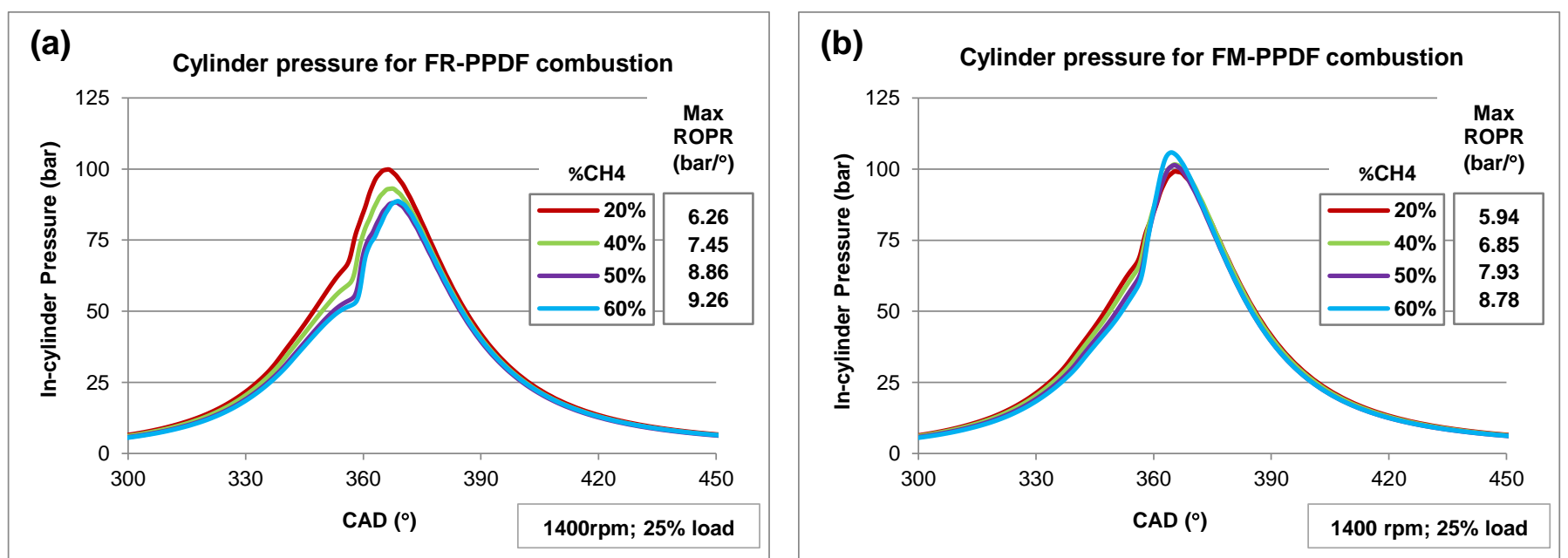

Fig. 2: In-cylinder pressure and the associated maximum ROPR; for (a) fixed ratio (FR) PPDF combustion, and (b) fixed mass (FM) PPDF combustion.

Figure 3 shows the net HRR, along with the corresponding positions of the occurrence of CA50; for FR-PPDF and FM-PPDF combustion. The influence of the reduction of the FR pre-injection mass as the $\% \mathrm{CH}_{4}$ is increased could be clearly noticed in Fig. 3 (a), where the reduction in the pre-injection mass results in a decay in the LTR zone; reducing the amount of the active radicals that promotes the combustion. Accordingly, the ability of the pre-injection to sustain a HTR (HTR-1) that could allow for the entrainment of some of the methane becomes very limited; especially with high substitution ratios. The resulting main combustion (HTR-2) therefore much resembles that of conventional DDF, where the ID and combustion duration (CD) both increase with the increase of $\% \mathrm{CH}_{4}$. With FM pre-injection, it is evident from Fig. 3 (b) that the magnitude of the LTR zone is almost the same, while it exhibits a slight shift towards the TDC as the $\% \mathrm{CH}_{4}$ increases. This could be attributed to the incidence of some chemical interactions between the methane and the diesel vapour [12]. All in all, the increased reactivity due to the cool flame reactions incorporate some of the methane in the combustion of the pre-injection part of the pilot; increasing the cylinder temperature and pressure [6]. The distinctive aspect of the process under these conditions is that ID almost remains constant for all $\% \mathrm{CH}_{4}$ employed; in contrast with FR-PPDF. Similarly, the duration of combustion is almost unchanged for all substitution ratios used. In view of that, it could be postulated that the effects of the increase of $\% \mathrm{CH}_{4}$ on elongating the ignition delay and combustion duration could be eliminated if a certain degree of charge reactivity was attained through the use of sufficient per-injection mass. The resulting intensified combustion takes place very rapidly during a few CAD [14], with the improved flame propagation at high $\% \mathrm{CH}_{4}$ brings the CA50 closer to the TDC. It is to the point here to highlight that for adiabatic engines, the optimal CA50 is attained just right at the TDC [10]. 

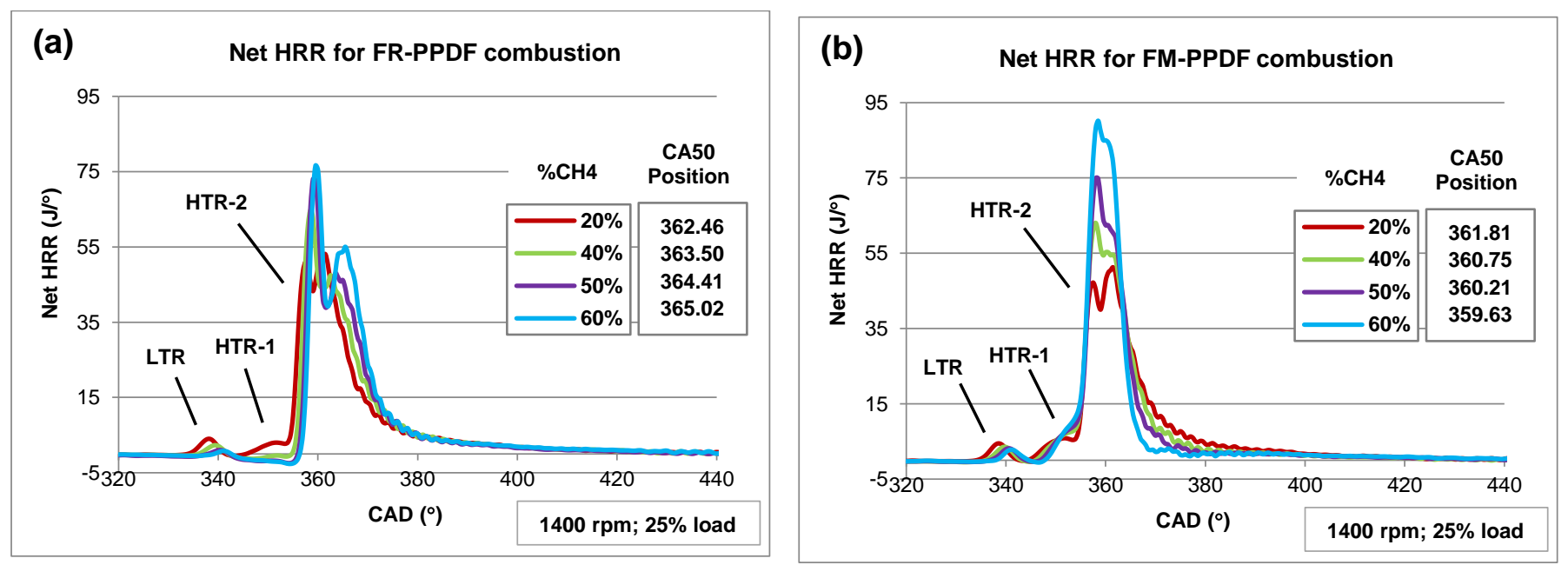

Fig. 3: Net HRR data, along with the corresponding positions of the occurrence of CA50; for (a) fixed ratio (FR) PPDF combustion, and (b) fixed mass (FM) PPDF combustion.

As far as the engine stability and performance are considered, it could be seen from Fig. 4 that FM-PPDF exhibits a slightly better engine stability (as represented by the lower value of $\operatorname{COV}_{\text {IMEPgross }}$ ), and also a slightly higher brake thermal efficiency; compared with FR-PPDF combustion. This slight improvement could be attributed to the higher in-cylinder pressure at the early stages of the expansion stroke, along with the reduced ROPR. Nevertheless, the values obtained with FR-PPDF are still very comparable. This is further demonstrated by Fig. 5 that presents the values of the total equivalence ratio $\left(\phi_{\mathrm{tot}}\right)$ and the total diesel fuel mass for both modes, where it could be seen that only minor reduction in the total fuel mass is attained with FM-PPDF compared with FR-PPDF. It is therefore expected to have a corresponding minor change in the PM emissions; yet the larger pre-injection mass is expected to increase the global mixture strength and reduce CO and $\mathrm{HC}$ emissions [4]. The reduced size of the main injection implies a corresponding reduction in the spray-cone rich mixture zone; potentially reducing NOx emissions in some measure [9].
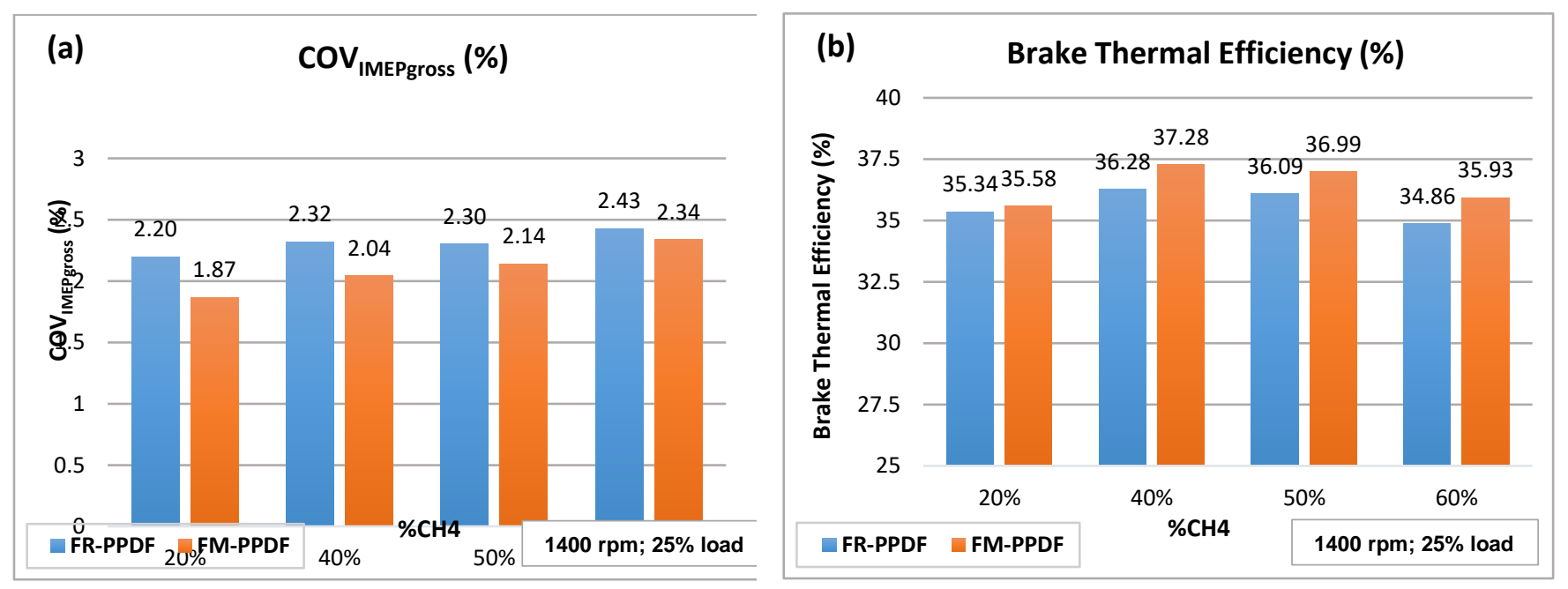

Fig. 4: (a) Coefficient of variation ( $\mathrm{COV}_{\text {IMEPgross }}$ ), and (b) brake thermal efficiency values; for FR-PPDF and FM-PPDF modes. 

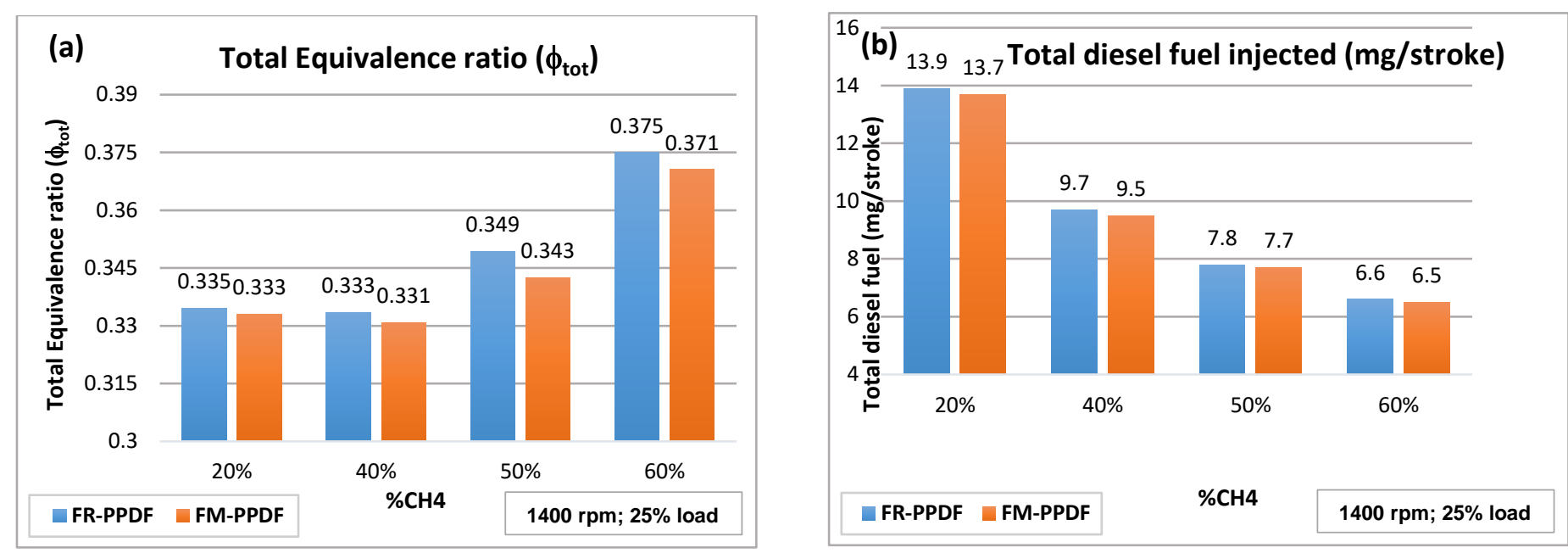

Fig. 5: (a) Total equivalence ratio ( $\phi_{\text {tot }}$ ), and (b) total diesel fuel injected (mg/stroke); for FR-PPDF and FM-PPDF modes.

\section{Conclusion}

The influence of pilot pre-injection mass on methane-diesel partially premixed dual fuel (PPDF) engine combustion was experimentally investigated; at part load conditions for different $\mathrm{CH} 4$ substitution ratios. The study compared two different pre-injection strategies; namely the fixed ratio (FR) and the fixed mass (FM). It was found that the decrease in pre-injection mass could bring about a decay in the LTR zone; reducing the amount of the active radicals that promotes the combustion. FM-PPDF exhibit higher cylinder pressure yet up to $8 \%$ lower values of maximum ROPR; compared with those obtained with FR-PPDF. Ignition delay and combustion duration both increase with the increase of $\% \mathrm{CH}_{4}$ with $\mathrm{FR}-$ PPDF, yet both almost remains unchanged for all substitution levels with FM-PPDF, where this was attributed to the attainment of a certain degree of charge reactivity through the use of sufficient per-injection mass. Engine stability and performance only improve slightly with FM-PPDF compared with FR-PPDF. While emissions results are not presented in the current work, it is expected to have lower CO and HC emissions with FM-PPDF, due to the increased mixture strength with pre-injection, and lower NOx and PM emissions due to the reduced size of the main injection; compared with FRPPDF.

\section{References}

[1] R. Bosch GmbH. Diesel-Engine Management. $4^{\text {th }}$ ed. West Sussex, UK: Wiley; 2006.

[2] Z. Wang, Z. Zhao, D. Wang, M. Tan, Y. Han, Z. Liu, and H. Dou, "Impact of pilot diesel ignition mode on combustion and emissions characteristics of a diesel/natural gas dual fuel heavy-duty engine," Fuel, vol. 167, pp. 248-256, 2016.

[3] G. A. Karim. Dual-Fuel Diesel Engines. Boca Raton, USA: CRC Press; 2015.

[4] F. Königsson, "On combustion in the CNG-diesel dual fuel engine," Ph.D. thesis, Dept. Mach. Des., Royal Institute of Technology (KTH), Sweden, 2014.

[5] M. Yao, Z. Zheng, and H. Liu, "Progress and recent trends in homogeneous charge compression ignition (HCCI) engines," Prog. Energy Combust. Sci., vol. 35, pp. 398-437, 2009.

[6] M. Konno, and Z. Chen, "Ignition mechanisms of HCCI combustion process fueled with methane/DME Composite Fuel," SAE Tech. Pap., 2005-01-0182, 2005.

[7] F. Königsson, P. Stålhammar, and H.-E. Ångström, “Combustion modes in a diesel-CNG dual fuel engine," $S A E$ Tech. Pap., 2011-01-1962, 2011.

[8] S. L. Kokjohn, R. M. Hanson, D. A. Splitter, and R. D. Reitz, "Experiments and modeling of dual-fuel HCCI and PCCI combustion using in-cylinder fuel blending," SAE Tech. Pap., 2009-01-2647, 2009.

[9] J. B. Heywood. Internal Combustion Engine Fundamentals. New York, USA: McGraw-Hill; 1988.

[10] L. de O. Carvalho, T. C. C. de Melo, and R. M. de A. C. Neto, "Investigation on the fuel and engine parameters that affect the half mass fraction burned (CA50) optimum crank angle," SAE Tech. Pap., 2012-36-0498, 2012.

[11] R. Stone. Introduction to Internal Combustion Engines. $3^{\text {rd }}$ ed., Oxford, UK: Macmillan; 1999. 
[12] Z. Liu, and G. A. Karim, "An examination of the ignition delay period in gas-fueled diesel engines," J. Eng. Gas Turbines Power, vol. 120, no. 1, pp. 225-231, 1998.

[13] M. Xu, W. Cheng, H. Zhang, T. An, S. Zhang, "Effect of diesel pre-injection timing on combustion and emission characteristics of compression ignited natural gas engine," Energy Convers. Manage., vol. 117, pp. 86-94, 2016.

[14] I. May, V. Pedrozo, H. Zhao, A. Cairns, S. Whelan, H. Wong, and P. Bennicke, "Characterization and potential of premixed dual-fuel combustion in a heavy duty natural gas/diesel engine," SAE Tech. Pap., 2016-01-0790, 2016. 